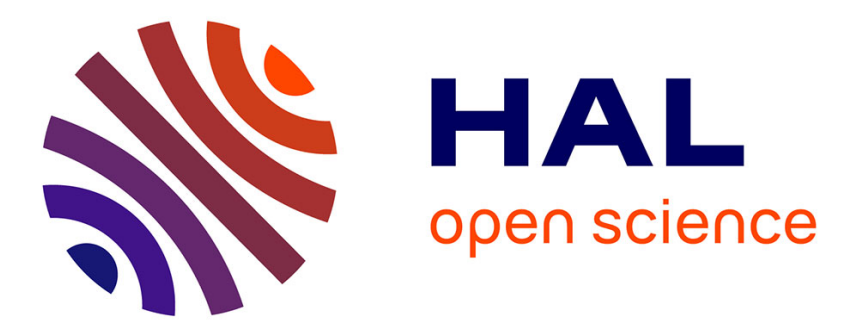

\title{
Etude de convoluteurs et corrélateurs acoustiques
}

\author{
Jean-Michel Rouvaen, G. Waxin, E. Bridoux, R. Torguet
}

\section{To cite this version:}

Jean-Michel Rouvaen, G. Waxin, E. Bridoux, R. Torguet. Etude de convoluteurs et corrélateurs acoustiques. Revue de Physique Appliquée, 1979, 14 (4), pp.575-586.

10.1051/rphysap:01979001404057500 . jpa-00244632

\section{HAL Id: jpa-00244632 \\ https://hal.science/jpa-00244632}

Submitted on 1 Jan 1979

HAL is a multi-disciplinary open access archive for the deposit and dissemination of scientific research documents, whether they are published or not. The documents may come from teaching and research institutions in France or abroad, or from public or private research centers.
L'archive ouverte pluridisciplinaire HAL, est destinée au dépôt et à la diffusion de documents scientifiques de niveau recherche, publiés ou non, émanant des établissements d'enseignement et de recherche français ou étrangers, des laboratoires publics ou privés. 


\title{
Etude de convoluteurs et corrélateurs acoustiques (*)
}

\author{
J. M. Rouvaen, G. Waxin, E. Bridoux et R. Torguet \\ Laboratoire d'Opto-acousto-électroniques $\left(^{(* *}\right)$ Centre Universitaire, 59326 Valenciennes Cedex, France
}

(Reçu le 25 septembre 1978, révisé le 22 décembre 1978, accepté le 2 janvier 1979)

\begin{abstract}
Résumé. - Des études antérieures ont montré l'existence d'une corrélation entre propriétés acousto-optiques et propriétés non linéaires élastiques : les matériaux possédant des facteurs de mérite acousto-optique élevés sont également très fortement non linéaires. Les interactions non linéaires dans ces matériaux ont été appliquées à la réalisations de nouveaux composants acoustiques permettant d'effectuer des fonctions de traitement du signal électronique classiques avec des rendements améliorés.
\end{abstract}

Abstract. - Some correlation between acousto-optic and nonlinear elastic properties has been found in previous studies. The non-linear elastic interactions in strongly photoelastic materials have thus been applied to the design of new acoustic electronic signal processing devices with improved efficiencies.

Introduction. - De nombreuses opérations de traitement du signal ont été réalisées à l'aide de processeurs optiques, qui peuvent être classés en processeurs cohérents ou incohérents.

L'avantage des processeurs optiques est leur temps de calcul très faible, fondamentalement égal au temps de propagation de la lumière entre les plans d'entrée et de sortie : un très grand nombre de signaux peut ainsi être traité à des cadences élevées. Cependant, un processeur optique est peu flexible. Le noyau de la transformation est la plupart du temps synthétisé au moyen d'une transparence optique réalisée sur une pellicule photographique. Comme un contrôle précis de l'épaisseur locale du film est technologiquement irréalisable, il est difficile d'obtenir une phase arbitrairement variable pour la transparence. Aussi se limite-t-on à des fonctions de transparence réelles et simples; à chaque opération correspondant une transparence différente. Il faut aussi remarquer que la quantité observable à la sortie d'un processeur optique est la distribution d'intensité lumineuse. Le détecteur final introduit alors toujours une nonlinéarité, le processus de détection n'obéissant que très imparfaitement à la loi quadratique ou linéaire qui serait souhaitable.

Les ondes acoustiques progressives constituent un moyen de stockage d'une \grande quantité d'informations (variant en fonction d'un paramètre unique).

$\left(^{*}\right)$ Etude réalisée dans le cadre de l'A.T.P. « Physique des dispositifs électroniques ".

(**) ERA 593 C.N.R.S
Un film photographique possède une capacité de stockage comparable, mais dans deux dimensions. Cependant, les informations sont figées et on obtient une mémoire statique.

Une mémoire dynamique est par contre obtenue avec les ondes acoustiques, les entrées variant en temps réel et possédant un spectre de fréquences assez large. Ceci est également vrai pour les lignes à retard électromagnétiques, mais le rapport de la vitesse de la lumière à celle des ondes acoustiques (en général de l'ordre de $10^{5}$ ) joue en faveur de ces dernières. Les lignes à retard acoustiques sont en effet beaucoup moins volumineuses et présentent moins de pertes.

Nous étudions ici des processus paramétriques basés sur l'interaction non linéaire entre des ondes acoustiques progressives.

Dans ces composants paramétriques non linéaires, un des signaux joue le rôle de la réponse impulsionnelle du filtre modifiable en temps réel : ils constituent donc des filtres électroniquement variables.

1. Généralités sur les interactions non linéaires. 1.1 EQUATIONS D'ÉTAT NON LINÉAIRES DES SOLIDES. - La théorie générale de la thermoélasticité $[1,2]$ établit les équations de propagation non linéaires ainsi que les conditions aux limites éventuelles sur un dioptre limitant le milieu, en se bornant aux termes non linéaires d'ordre le plus faible, c'est-à-dire quadratiques par rapport au déplacement mécanique $\mathbf{u}$.

En utilisant la convention de sommation d'Einstein ainsi que les notations indicielles $J, J$ et ${ }^{~}{ }^{\text {pour repré- }}$ 
senter, respectivement, les opérateurs $\partial / \partial x_{j}$ et $\partial^{2} / \partial t^{2}$, nous pouvons écrire, dans le cas d'un milieu non piézoélectrique :

$$
\begin{aligned}
& \rho \ddot{u}_{\mathrm{M}}=C_{\mathrm{MNPQ}} u_{\mathrm{P}, \mathrm{NQ}}+C_{\mathrm{MNPQRS}}^{\mathrm{F}} u_{\mathrm{P}, \mathrm{NQ}} u_{\mathrm{R}, \mathrm{S}} \\
& \text { (équations du mouvement) } \\
& T_{\mathrm{MN}}=C_{\mathrm{MNPQ}} u_{\mathrm{P}, \mathrm{Q}}+C_{\mathrm{MNPQRS}}^{\mathrm{S}} u_{\mathrm{P}, \mathrm{Q}} u_{\mathrm{R}, \mathrm{S}} \\
& \text { (contraintes non linéaires) }
\end{aligned}
$$

où $\rho$ représente la masse volumique du milieu au repos (en l'absence d'onde élastique), $C_{\mathrm{MNPQ}}$ est une constante élastique du second ordre et $C_{\mathrm{MNPQRS}}^{\mathrm{F}, \mathrm{S}}$ sont des constantes élastiques effectives du troisième ordre, données par des combinaisons linéaires de constantes élastiques des second et troisième ordres :

$$
\begin{aligned}
& C_{\mathrm{MNPQRS}}^{\mathrm{F}}=C_{\mathrm{MNPQRS}}+\delta_{\mathrm{MP}} C_{\mathrm{NQRS}}+ \delta_{\mathrm{PR}} C_{\mathrm{MNQS}}+ \\
&+\delta_{\mathrm{MR}} C_{\mathrm{NSPQ}} \\
& C_{\mathrm{MNPQRS}}^{\mathrm{S}}=\frac{1}{2} C_{\mathrm{MNPQRS}}+\frac{1}{2} \delta_{\mathrm{PR}} C_{\mathrm{MNQS}}+\delta_{\mathrm{MR}} C_{\mathrm{NSPQ}}
\end{aligned}
$$

où $\delta$ est un symbole de Kronecker (élément de la matrice unité de rang 3 ).

1.2 DESCRIPTION GÉNÉRALE D'UN PHÉNOMÈNE D'INTERACTION A TROIS ONDES ÉLASTIQUES. - Le champ de déplacement total $\mathbf{u}$ est, en première approximation, la superposition des champs élastiques primaires désignés ici par des indices supérieurs 1 et 2 :

$$
\mathbf{u}=\mathbf{u}^{1}+\mathbf{u}^{2}+\text { termes d'ordre supérieur. }
$$

Nous effectuons ainsi l'approximation de la propagation linéaire pour les ondes primaires, dont nous supposons qu'elles ne sont pas affectées de façon sensible par l'interaction non linéaire. Ceci ne constitue pas une simplification excessive du problème car, dans les matériaux utilisés en microacoustique, l'interaction non linéaire demeure faible en ellemême, mais des effets spectaculaires peuvent néanmoins être obtenus du fait du caractère cumulatif de certaines interactions.

En reportant la solution (5) dans les équations (1) et (2), nous constatons que les champs élastiques primaires donnent naissance à des densités volumiques de force non linéaires et à des contraintes non linéaires, parmi lesquelles celles correspondant à la génération d'ondes harmoniques (interaction de $\mathbf{u}^{1}$ ou $\mathbf{u}^{2}$ avec lui-même) seront négligées ici. Nous ne retenons donc que les termes provenant de l'interaction non linéaire entre les deux champs élastiques primaires dans leur zone de recouvrement (région d'interaction), qui consistent en une densité volumique de forces non linéaires non équilibrée :

$$
F_{\mathrm{M}}^{\mathrm{NL}}=C_{\mathrm{MNPQRS}}^{\mathbf{F}}\left(u_{\mathrm{P}, \mathrm{NQ}}^{1} u_{\mathrm{R}, \mathrm{S}}^{2}+u_{\mathrm{P}, \mathrm{NQ}}^{2} u_{\mathrm{R}, \mathrm{S}}^{1}\right)
$$

et en des contraintes non linéaires non compensées :

$$
T_{\mathrm{MN}}^{\mathrm{NL}}=C_{\mathrm{MNPQRS}}^{\mathrm{S}}\left(u_{\mathrm{P}, \mathrm{Q}}^{1} u_{\mathrm{R}, \mathrm{S}}^{2}+u_{\mathrm{P}, \mathrm{Q}}^{2} u_{\mathrm{R}, \mathrm{S}}^{1}\right)
$$

Les termes d'ordre supérieur de l'équation (5) constituent alors un champ de déplacement mécanique diffracté $\mathbf{u}^{3}$, engendré par interaction non linéaire, équilibrant les termes sources (6) et (7) précédents dans la région d'interaction.

Le champ diffracté $\mathbf{u}^{3}$ est donc obtenu en résolvant les équations du mouvement forcé :

$$
\rho \ddot{u}_{\mathrm{M}}^{3}=C_{\mathrm{MNPQ}} u_{\mathrm{P}, \mathrm{NQ}}^{3}+F_{\mathrm{M}}^{\mathrm{NL}}
$$

en tenant éventuellement compte des conditions aux limites du problème (qui font intervenir les contraintes non linéaires $T_{\mathrm{MN}}^{\mathrm{NL}}$ ). En général, le champ diffracté ne correspond pas à des modes normaux élastiques de propagation du milieu dépourvu de sources (c'est-à-dire linéaire, comme c'est le cas en dehors de la région d'interaction). On observe alors le rayonnement d'ondes rapidement évanescentes vers l'extérieur de la région d'interaction. Par contre, en choisissant correctement les polarisations et les fréquences des ondes primaires, une partie du champ diffracté peut coïncider avec les modes normaux précédents, d'où l'émission vers l'extérieur de la région d'interaction d'ondes progressives élastiques. On a alors une interaction synchrone produisant une onde diffractée observable, ce qui suggère, pour la résolution du problème, une décomposition du champ diffracté selon les modes normaux de propagation libre.

Considérons alors des champs primaires harmoniques :

$$
\mathbf{u}^{i}=\frac{1}{2} \mathbf{a}^{i} \exp \left(j \omega_{i} t\right)+\text { C.C. } \quad(i=1,2)
$$

où les $\omega_{i}$ sont les pulsations de ces champs, C.C. désigne la quantité complexe conjuguée, et les vecteurs $\mathbf{a}_{i}$ sont donnés par :

$$
\mathbf{a}^{i}=\mathbf{b}^{i} \exp \left(-j \omega_{i} \mathbf{s}^{i} \mathbf{r}\right)=\mathbf{b}^{i} \exp \left(-j \omega_{i} s_{i} \mathbf{N}^{i} \mathbf{r}\right)
$$

où $\mathbf{r}$ est le rayon vecteur joignant l'origine au point d'observation, les $\mathbf{b}^{i}$ décrivent les amplitudes des champs primaires et peuvent être fonctions des coordonnées spatiales et du temps, les $s_{i}$ sont les lenteurs (inverses des vitesses de phase) des ondes primaires, dont les vecteurs $\mathbf{N}^{i}$ fixent les directions de propagation $\left(\mathbf{s}^{i}=s_{i} \mathbf{N}^{i}\right)$.

D'après les équations (6) à (8), le champ diffracté peut se mettre sous la forme d'une somme de termes de fréquence somme et de fréquence différence : $\mathbf{u}^{3}=\frac{1}{2} \mathbf{a}_{+}^{3} \exp j\left(\omega_{1}+\omega_{2}\right) t+$

$$
+\frac{1}{2} \mathbf{a}_{-}^{3} \exp j\left(\omega_{1}-\omega_{2}\right) t+\text { C.C. }
$$

correspondant à des termes sources de fréquence somme et de fréquence différence.

Les variations de phase du champ diffracté doivent être identiques à celles des termes sources correspondants afin que l'interaction puisse être synchrone. Ceci conduit aux conditions de synchronisme :

$$
\begin{gathered}
\left(\omega_{3}\right)_{ \pm}=\omega_{1} \pm \omega_{2} \\
\left(\omega_{3}\right)_{ \pm} \mathbf{s}_{ \pm}^{3}=\omega_{1} s^{1} \pm \omega_{2} s^{2}
\end{gathered}
$$


pour les fréquences somme et différence, où nous avons introduit le vecteur de lenteur diffracté $\mathbf{s}^{3}$ et supposé $\omega_{1}>\omega_{2}$.

On voit qu'il s'agit en fait de conditions de conservation pour la fréquence et pour le vecteur d'onde $\mathbf{k}=\omega \mathbf{s}$.

1.3 ETUDE DES INTERACTIONS COLINÉAIRES. - Nous avons jusqu'ici considéré le cas tout à fait général où les champs primaires se propagent suivant des directions différentes, de même que le champ diffracté. Nous obtenons ainsi des interactions non colinéaires.

Un cas particulier important est celui des interactions colinéaires où tous les champs se propagent suivant une direction unique, ce qui a pour effet de simplifier grandement les équations. En particulier, dans les conditions de synchronisme (12) et (13), les vecteurs de lenteur deviennent des nombres algébriques.

Lorsque les lenteurs des champs acoustiques primaires et diffractés sont identiques, il y a dégénérescence : les ondes élastiques interagissantes ont toutes même polarisation et même sens, de sorte que les conditions de synchronisme sont identiquement vérifiées pour des valeurs arbitraires des pulsations primaires $\omega_{1}$ et $\omega_{2}$ (avec toutefois par hypothèse $\left.\omega_{1}>\omega_{2}\right)$.

Nous exclurons ce cas dans ce qui suit en considérant des ondes élastiques interagissantes de polarisations non toutes identiques et en envisageant le cas particulier important des ondes élastiques de volume.

Les ondes élastiques planes de volume sont non dispersives, c'est-à-dire que leurs lenteurs sont indépendantes de leurs pulsations. De plus, dans ce cas, les vecteurs $\mathbf{b}^{i}$ de l'équation (10) sont proportionnels aux vecteurs unitaires de polarisation acoustique $\mathbf{U}[3,4,5]$.

$$
\mathbf{b}^{i}=A_{i} \mathbf{U}^{i} \quad(i=1,2)
$$

où les $A_{i}$ sont les amplitudes complexes des ondes primaires, pouvant être fonctions progressives du temps et de la coordonnée spatiale $x_{1}$ :

$$
A_{i}=A_{i}\left(t-s_{i} x_{1}\right)
$$

si le repère de travail est choisi de façon que l'axe $x_{1}$ précédent coïncide avec la direction de propagation commune des ondes primaires et diffractée.

Les ondes planes étant supposées d'extension infinie dans le plan $\left(x_{2}, x_{3}\right)$, aucune condition aux limites n'est alors à considérer et il suffit de tenir compte des termes sources qui s'écrivent :

$$
\begin{aligned}
&\left(F_{\mathbf{M}}^{\mathrm{NL}}\right)_{ \pm}=\frac{ \pm \mathrm{j} \omega_{1} \omega_{2}\left(\omega_{1} s_{1} \pm \omega_{2} s_{2}\right) s_{1} s_{2} A_{1} A_{2}^{*}}{4} \times \\
& \times C_{\mathbf{M} 1 \mathbf{P} 1 \mathbf{R} 1}^{\mathbf{F}} U_{\mathbf{P}}^{1} U_{\mathbf{R}}^{2} \exp \left[-j \ldots\left(\omega_{1} s_{1} \pm \omega_{2} s_{2}\right) x_{1}\right] \\
& \times \exp j\left(\omega_{1} \pm \omega_{2}\right) t
\end{aligned}
$$

où le signe * désigne l'amplitude elle-même dans le cas de la fréquence somme et sa complexe conjuguée dans le cas de la fréquence différence.

L'onde diffractée peut être décomposée selon les vecteurs de polarisation acoustique des ondes élastiques de volume pouvant se propager selon la direction $x_{1}$. On sait qu'il s'agit de trois vecteurs mutuellement orthogonaux et formant une base $[3,4,5]$. Nous pouvons alors écrire :

$$
\begin{aligned}
& \mathbf{u}_{ \pm}^{3}=\frac{1}{3} A_{\alpha}^{ \pm}\left(x_{1}, t\right) \mathbf{U}^{\alpha} \exp \left[j \omega_{3}\left(t-s_{\alpha} x_{1}\right)\right]+ \\
& +\frac{1}{2} A_{\beta}^{ \pm}\left(x_{1}, t\right) \mathbf{U}^{\beta} \exp \left[j \omega_{3}\left(t-s_{\beta} x_{1}\right)\right] \\
& +\frac{1}{2} A_{\gamma}^{ \pm}\left(x_{1}, t\right) \mathbf{U}^{\gamma} \exp \left[j \omega_{3}\left(t-s_{\gamma} x_{1}\right)\right]+\text { C.C. }
\end{aligned}
$$

où $\left(\mathbf{U}^{\alpha}, \mathbf{U}^{\beta}, \mathbf{U}^{\gamma}\right)$ représente la base précédente, les amplitudes $A_{\alpha}^{ \pm}, A_{\beta}^{ \pm}$et $A_{\gamma}^{ \pm}$étant supposées fonctions lentement variables de la coordonnée $x_{1}$ et du temps.

Compte tenu des équations de Christoffel pour les ondes élastiques $[3,4,5]$, nous pouvons écrire :

$$
C_{\mathbf{M} 1 \mathbf{P} 1} U_{\mathbf{P}}^{\varepsilon} s_{\varepsilon}^{2}=\rho U_{\mathbf{M}}^{\varepsilon}
$$

pour une polarisation $\varepsilon$ quelconque, de sorte que l'équation (8) compte tenu de l'orthogonalité des vecteurs de polarisation, devient :

$\left(\frac{\partial}{\partial x_{1}}+s_{\varepsilon} \frac{\partial}{\partial t}\right) A_{\varepsilon}^{ \pm}=\frac{\mathrm{d} A_{\varepsilon}^{ \pm}}{\mathrm{d} x_{1}}=\lambda_{ \pm}^{\gamma} A_{1} A_{2}^{*} \exp \left(j \varphi_{\varepsilon}^{ \pm}\right)$

où nous avons posé :

$$
\varphi_{\varepsilon}^{ \pm}=\left[\left(\omega_{1} \pm \omega_{2}\right) s_{\varepsilon}-\left(\omega_{1} s_{1} \pm \omega_{2} s_{2}\right)\right] x_{1} .
$$

Pour une interaction donnée, à la fréquence somme ou à la fréquence différence, l'accord de phase peut être obtenu pour l'onde de polarisation $\varepsilon$ lorsque le déphasage $\varphi_{\varepsilon}^{ \pm}$est nul, soit lorsque les conditions de synchronisme sont satisfaites pour cette onde. L'onde correspondante va croître cumultativement au cours de l'interaction, pour peu que le coefficient de couplage non linéaire :

$\lambda_{ \pm}^{\varepsilon}=\frac{ \pm \omega_{1} \omega_{2}\left(\omega_{1} s_{1} \pm \omega_{2} s_{2}\right) s_{\varepsilon} s_{1} s_{2} C_{\mathrm{M} 1 \mathrm{P} 1 \mathrm{R} 1}^{\mathbf{F}} U_{\mathrm{M}}^{\varepsilon} U_{\mathrm{P}}^{1} U_{\mathrm{R}}^{2}}{4 \rho\left(\omega_{1} \pm \omega_{2}\right)}$

ne soit pas identiquement nul, auquel cas l'interaction n'a pas lieu. Ceci fait apparaître la notion de règles de sélection donnant les interactions possibles pour deux ondes élastiques primaires de polarisations données.

Les deux autres ondes, non adaptées aux termes sources, vont effectuer des oscillations périodiques sans jamais atteindre des valeurs importantes : on peut les négliger en pratique dans la plupart des cas. Nous obtenons ainsi une interaction à trois ondes, 
l'amplitude $A_{3}$ de l'onde engendrée au synchronisme étant donnée par l'équation :

$\frac{\mathrm{d} A_{3}^{ \pm}}{\mathrm{d} x_{1}}= \pm \omega_{1} \omega_{2} A_{1} A_{2}^{*} \frac{C_{\mathrm{M} 1 \mathrm{P} 1 \mathrm{R} 1}^{\mathrm{F}} U_{\mathrm{M}}^{3} U_{\mathrm{P}}^{1} U_{\mathrm{R}}^{2} s_{3}^{2}}{4 \rho} s_{1} s_{2}$

expression analogue à celles données dans un certain nombre de travaux antérieurs $[6,7,8,9]$.

2. Traitement du signal au moyen d'une interaction paramétrique entre trois ondes élastiques de volume colinéaires. - 2.1 ONDES PARTICIPANT A L'INTERACTION. - Considérons un milieu non linéaire et une direction principale de ce dernier selon laquelle peuvent se propager trois ondes élastiques pures (c'est-à-dire telles que les vitesses de propagation de l'énergie et de la phase soient colinéaires). Ces ondes sont une onde longitudinale de vitesse $v_{\mathrm{L}}$ et deux ondes transversales de vitesses $v_{\mathrm{T} 1}$ et $v_{\mathrm{T} 2}$ et éventuellement égales (on dit alors qu'il y a dégénérescence). Ceci est vérifié pour une direction de propagation quelconque dans le cas d'un milieu isotrope et pour certains axes de symétrie particuliers dans le cas des milieux monocristallins [4].

En considérant alors les constantes élastiques du troisième ordre non nulles [10], on montre que seules sont observables les interactions faisant intervenir une onde longitudinale et deux ondes transversales, polarisées dans la même direction, dès qu'il y a dégénérescence (axe de symétrie d'ordre 4 ou 6 , ou milieu isotrope), ou encore trois ondes longitudinales. Seul le premier cas fait intervenir des ondes élastiques de lenteurs différentes, condition nécessaire pour réaliser un traitement du signal, comme nous le verrons plus bas.

On obtient un dispositif non linéaire utilisant une onde longitudinale et deux ondes transversales dégénérées, deux quelconques de ces ondes transportant les signaux d'entrée et la troisième transportant le signal de sortie.

2.2 Conditions De SYNCHRONISME. - En désignant par $\omega_{\mathrm{L}}, \omega_{\mathrm{T} 1}$ et $\omega_{\mathrm{T} 2}$ les pulsations des trois ondes interagissantes, avec sans perte de généralité $\omega_{\mathrm{T} 1}>\omega_{\mathrm{T} 2}$, les conditions de synchronisme pour l'obtention d'une interaction non linéaire constructive s'écrivent :

$$
\begin{gathered}
\omega_{\mathrm{L}}+\alpha_{1} \omega_{\mathrm{T} 1}+\alpha_{2} \omega_{\mathrm{L} 2}=0 \\
\omega_{\mathrm{L}} s_{\mathrm{L}}+\alpha_{1} \omega_{\mathrm{T} 1} s_{\mathrm{T} 1}+\alpha_{2} \omega_{\mathrm{T} 2} s_{\mathrm{T} 2}=0
\end{gathered}
$$

où les lenteurs (inverses des vitesses) des ondes interagissantes sont données par :

$$
s_{\mathrm{L}}=1 / v_{\mathrm{L}}
$$

(le sens de propagation de l'onde longitudinale étant arbitrairement pris positif).

$$
s_{\mathrm{T} 1}=\varepsilon_{1} / v_{\mathrm{T}}
$$

$$
\begin{gathered}
s_{\mathrm{T} 2}=\varepsilon_{2} / v_{\mathrm{T}} \\
\alpha_{1}, \alpha_{2}, \varepsilon_{1}, \varepsilon_{2}= \pm 1 .
\end{gathered}
$$

La condition $\frac{\omega_{\mathrm{T} 1}}{\omega_{\mathrm{L}}}>\frac{\omega_{\mathrm{T} 2}}{\omega_{\mathrm{L}}}>0$ montre que la seule solution possible correspond à :

$$
\alpha_{1}=\alpha_{2}=-1 ; \quad \varepsilon_{1}=-\varepsilon_{2}=1 .
$$

La fréquence de l'onde longitudinale est donc égale à la somme de celles des ondes transversales. De plus, l'onde transversale de plus haute fréquence se propage dans le même sens que l'onde longitudinale, alors que l'autre se propage en sens opposé. Le synchronisme est donc obtenu pour des valeurs particulières suivantes des rapports de pulsations :

$$
\begin{aligned}
& \frac{\omega_{\mathrm{T} 1}}{\omega_{\mathrm{L}}}=\frac{v_{\mathrm{L}}+v_{\mathrm{T}}}{2 v_{\mathrm{L}}} \\
& \frac{\omega_{\mathrm{T} 2}}{\omega_{\mathrm{L}}}=\frac{v_{\mathrm{L}}-v_{\mathrm{T}}}{2 v_{\mathrm{L}}}
\end{aligned}
$$

2.3 INTERACTION PARAMÉTRIQUE EN RÉGIME NON STATIONNAIRE. - L'équation non linéaire du mouvement, dans l'approximation des amplitudes lentement variables dans l'espace (à l'échelle de la longueur d'onde acoustique) et dans le temps (à l'échelle de la période acoustique) fournit l'amplitude $\mathrm{d} a_{0}$ de l'ondelette élémentaire engendrée au cours de l'interaction à l'abscisse $x$ et au temps $t$ :

$$
\mathrm{d} a_{0}(x, t)=\gamma a_{1}(x, t) a_{2}(x, t) \exp (j \Delta k x) \mathrm{d} x
$$

où $\gamma$ est un coefficient de couplage non linéaire, $a_{1}(x, t)$ et $a_{2}(x, t)$ représentent les modulations d'amplitude des ondes élastiques primaires, $\Delta k$ représente un désaccord de phase pour les vecteurs d'onde par rapport au synchronisme et $\mathrm{d} x$ représente la longueur d'un tronçon infinitésimal du milieu élastique. En désignant par $s_{\mathrm{i}}$ les lenteurs algébriques des deux ondes primaires et $x_{0 \mathrm{i}}$ les abscisses auxquelles ces ondes sont produites $(i=1,2)$, nous avons :

$$
a_{i}(x, t)=\eta_{i} f_{i}\left[t-s_{i}\left(x-x_{0 i}\right)\right]
$$

où $f_{i}(t)$ représente l'amplitude (lentement variable) de la tension électrique excitant le transducteur piézoélectrique, de facteur de conversion acoustoélectrique $\eta_{i}$, qui excite l'onde primaire.

Toutes les ondelettes émises en divers points et à divers instants se propagent avec la lenteur algébrique $s_{0}$ vers un transducteur piézoélectrique récepteur situé à l'abscisse $X$. Les ondelettes arrivant sur le transducteur récepteur à l'instant $T$ sont celles qui ont été émises aux abscisses $x$ et aux instants $t$ tels que :

$$
T-t=s_{0}(X-x)
$$


de sorte que le signal électrique de sortie, délivré par le transducteur est de la forme :

$$
S_{X}(T)=\eta_{0} \int_{\text {milieu }} \mathrm{d} a_{0}\left[x, T-s_{0}(X-x)\right]
$$

où $\eta_{0}$ est le facteur de conversion électroacoustique.

Nous pouvons écrire :

$$
\begin{aligned}
S_{X}(T)= & \eta_{0} \eta_{1} \eta_{2} \gamma \int_{\text {milieu }} f_{1}\left[T-\tau_{1}+\left(s_{0}-s_{1}\right) x\right] \times \\
& \times f_{2}\left[T-\tau_{2}+\left(s_{0}-s_{2}\right) x\right] \exp (j \Delta k x) \mathrm{d} x
\end{aligned}
$$

où nous avons posé :

$$
\tau_{i}=s_{0} X-s_{i} x_{0 i} .
$$

Si nous supposons la durée des signaux $f_{i}$ limitée dans le temps et inférieure au temps de propagation dans le milieu, les bornes d'intégration dans l'équation (35) peuvent être considérées comme infinies.

Lorsque les conditions de synchronisme ne sont pas satisfaites $(\Delta k \neq 0)$, l'équation (35) représente une généralisation de la fonction d'ambiguité, définie mathématiquement pour deux signaux $f_{1}(u)$ et $f_{2}(u)$ comme :

$$
\begin{aligned}
\psi_{12}(v, \Delta \omega) & = \\
= & \int_{-\infty}^{+\infty} f_{1}(u) f_{2}(u-v) \exp (j \Delta \omega u) \mathrm{d} u
\end{aligned}
$$

qui représente l'incertitude de localisation par les techniques radar de cibles situées à des distances différentes et animées de vitesses différentes [11].

On voit, d'après cette définition, qu'aucune restriction n'est à faire dans le cas d'un produit du type corrélation, alors qu'une fonction d'ambiguité n'est obtenue en toute rigueur, dans le cas d'un produit du type convolution, que si le signal $f_{2}$ est symétrique dans le temps $\left(f_{2}(-t)=f_{2}(t)\right)$.

La fonction d'ambiguité a d'ailleurs déjà pu être obtenue dans le cas de signaux de durée temporelle limitée au moyen de convoluteurs classiques à ondes piézoélectriques de surface [12]. D'autre part, si l'un des signaux primaires est constant (ce qui correspond à une onde élastique primaire sinusoïdale entretenue), la fonction d'ambiguité précédente se ramène à une simple transformée de Fourier. En effet, en posant :

$$
\begin{aligned}
& \left(s_{0}-s_{1}\right) \omega=\Delta k \\
& D=\frac{\eta_{0} \eta_{1} \eta_{2} \gamma}{\left|s_{0}-s_{1}\right|} \exp \left(j \omega \tau_{1}\right) \\
& u=T-\tau_{1}+\left(s_{0}-s_{1}\right) x
\end{aligned}
$$

l'équation (35) s'écrit :

$$
F_{1}(\omega, T)=D \exp (-j \omega T) \int_{-\infty}^{+\infty} f_{1}(u) \exp (j \omega u) \mathrm{d} u .
$$

La transformée de Fourier du signal $f_{1}$ peut donc être obtenue en échantillonnant le signal de sortie de façon à conserver une valeur constante du paramètre $T$, tout en faisant varier $\omega$ (c'est-à-dire $\Delta k$ ) par l'intermédiaire de la fréquence de l'onde primaire 2 .

2.4 Transformation bilinéaire. - Plaçons-nous dans les conditions de synchronisme $(\Delta k=0)$ et effectuons le changement de variable :

$$
u=\tau_{2}+\left(s_{2}-s_{0}\right)\left(x+\frac{T}{s_{0}-s_{1}}\right) .
$$

L'équation (35) se met alors sous la forme d'une transformée bilinéaire :

$$
S_{X}(T)=C \int_{-\infty}^{+\infty} f_{1}(\alpha u+\beta) f_{2}(\gamma T-u) \mathrm{d} u
$$

avec :

$$
\begin{aligned}
& \alpha=\frac{s_{0}-s_{1}}{s_{2}-s_{0}} \\
& \beta=-\left(\tau_{1}+\alpha \tau_{2}\right) \\
& \gamma=1+\frac{1}{\alpha} \\
& C=\frac{\eta_{0} \eta_{1} \eta_{2} \gamma}{\left|s_{2}-s_{0}\right|} .
\end{aligned}
$$

Les propriétés de la transformation bilinéaire générale sont discutées en annexe.

La relation (46) montre que deux cas sont à considérer :

- si $\alpha>0$ (et donc $\gamma>0$ ) l'intégrale (43) est une généralisation du produit de convolution;

- si $\alpha<0$ il s'agit d'une généralisation du produit de corrélation.

D'autre part, la numérotation des ondes primaires étant arbitraire, l'échange des rôles des ondes 1 et 2 revient en fait principalement à transformer $\alpha$ en son inverse $1 / \alpha$.

En utilisant les résultats de l'annexe, nous obtenons les paramètres de l'interaction dans les trois cas possibles, donnés au tableau I.

La condition d'obtention d'un signal de sortie triangulaire en réponse à deux impulsions rectangulaires (A.13) s'écrit alors toujours sous la forme :

$$
\omega_{1} t_{1}=\omega_{2} t_{2}
$$

où $t_{1}$ et $t_{2}$ sont les durées des impulsions.

Ceci implique une condition de conservation du nombre de périodes acoustiques : les deux impulsions primaires comprennent en effet, vu la condition (48) un nombre égal de périodes et la largeur maximale du signal de sortie triangulaire (à la base), en comprend le double.

Nous constatons enfin que, dans les deux premiers cas du tableau I, le signal de sortie est un produit 
de corrélation et sa fréquence est égale à la différence de celles des signaux d'entrée. Dans le dernier cas, le signal de sortie, de fréquence égale à la somme de celles des signaux d'entrée, est un produit de convolution. Un même dispositif permet ainsi, par simple permutation des entrées et de la sortie, d'obtenir deux produits de corrélation et un produit de convolution.

Tableau I. - Echelle de temps pour les interactions paramétriques à trois ondes.

[Time scale for three waves parametric interactions.]

$\begin{array}{cccc}\text { Onde 1 } & \text { Onde 2 } & \text { Onde } 0 & \alpha \\ - & - & - & - \\ \mathrm{L} & \mathrm{T} 1 & \mathrm{~T} 2 & -\left(v_{\mathrm{L}}+v_{\mathrm{T}}\right) / 2 v_{\mathrm{L}}=-\omega_{\mathrm{T} 1} / \omega_{\mathrm{L}} \\ \mathrm{L} & \mathrm{T} 2 & \mathrm{~T} 1 & -\left(v_{\mathrm{L}}-v_{\mathrm{T}}\right) / 2 v_{\mathrm{L}}=-\omega_{\mathrm{T} 2} / \omega_{\mathrm{L}} \\ \mathrm{T} 1 & \mathrm{~T} 2 & \mathrm{~L} & \left(v_{\mathrm{L}}-v_{\mathrm{T}}\right) /\left(v_{\mathrm{L}}+v_{\mathrm{T}}\right)=\omega_{\mathrm{T} 2} / \omega_{\mathrm{T} 1}\end{array}$

3. Etude détaillée d'un dispositif de traitement du signal par interaction non linéaire élastique. - 3.1 Produit de CONvolution. - Nous avons vu que le produit de convolution est obtenu en utilisant deux ondes primaires transversales et une onde de sortie longitudinale à la fréquence somme. L'interaction est alors symbolisée par la figure 1 où nous avons supposé le récepteur situé à l'abscisse $X=L$.

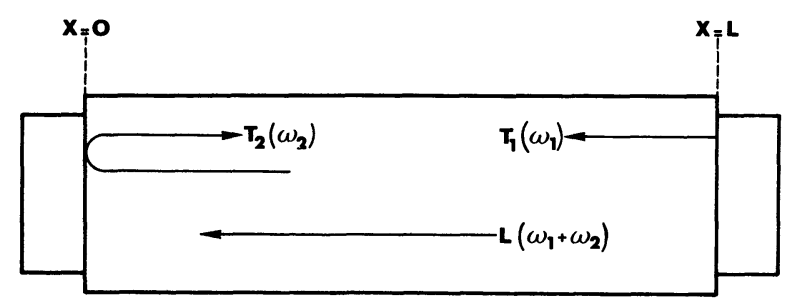

Fig. 1. - Dispositif de convolution $\left(\omega_{1}>\omega_{2}\right)$.

[Convolution device $\left(\omega_{1}>\omega_{2}\right)$.]

Les relations (36) et (45) s'écrivent alors :

$$
\begin{aligned}
& \tau_{1}=-\beta=L / v_{\mathrm{T}}=T_{\mathrm{M}} \\
& \tau_{2}=0
\end{aligned}
$$

car, vu les dimensions finies du milieu non linéaire, le temps maximum de traitement est celui $L / v_{\mathrm{T}}$ mis par une onde transversale pour le parcourir dans toute sa longueur. Il convient de remarquer que le signal $T_{2}$ doit alors être, afin de bénéficier d'un tel temps de traitement, émis avec cette même avance $T_{\mathrm{M}}$ par rapport au signal $T_{1}$.

La relation (47) donne :

$$
C=\frac{\eta_{0} \eta_{1} \eta_{2} \gamma v_{\mathrm{L}} v_{\mathrm{T}}}{v_{\mathrm{L}}-v_{\mathrm{T}}}
$$

L'étude du noyau de la transformation dans l'espace des fréquences s'effectue selon la méthode générale de l'annexe. Les résultats sont analogues à ceux des équations (A.14) à (A.23).

En particulier, si le temps de traitement devient grand, le noyau n'est non nul que si le paramètre $x_{0}$ de l'équation (A.19) est nul (avec $A=T_{\mathbf{M}}$ ). Ceci implique ici :

$$
\frac{\omega_{2}}{\omega_{1}}=\frac{v_{\mathrm{L}}-v_{\mathrm{T}}}{v_{\mathrm{L}}+v_{\mathrm{T}}}=\frac{\omega_{\mathrm{T} 2}}{\omega_{\mathrm{T} 1}}
$$

et nous retrouvons en fait la condition de synchronisme pour les ondes primaires.

3.2 Produit de CORRÉlation. - Nous avons vu que les produits de corrélation sont obtenus en utilisant une onde primaire longitudinale et une onde primaire transversale, l'onde de sortie étant une seconde onde transversale, de fréquence égale à celle de l'onde longitudinale diminuée de celle de l'onde transversale primaire. Le premier cas, où les ondes primaires se propagent en sens opposés, est symbolisé par la figure 2 .

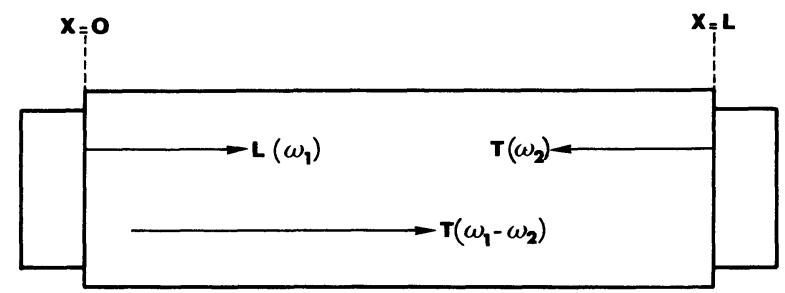

Fig. 2. - Premier cas de corrélation $\left(\omega_{1}>2 \omega_{2}\right)$.

[First correlation device $\left(\omega_{1}>2 \omega_{2}\right)$.]

Les relations (36), (45) et (47) s'écrivent :

$$
\begin{gathered}
\tau_{1}=\tau_{2} / 2=L / v_{\mathrm{T}}=T_{\mathrm{M}} \\
2 \beta v_{\mathrm{L}}=-\left(v_{\mathrm{L}}+v_{\mathrm{T}}\right) \tau_{1} \\
C=\frac{\eta_{0} \eta_{1} \eta_{2} \gamma v_{\mathrm{T}}}{2}
\end{gathered}
$$

(le temps de traitement maximal a toujours la même valeur $T_{\mathrm{M}}$ que pour la convolution).

Dans le second cas de corrélation, les ondes primaires se propagent dans le même sens, d'où la représentation symbolique de la figure 3 .

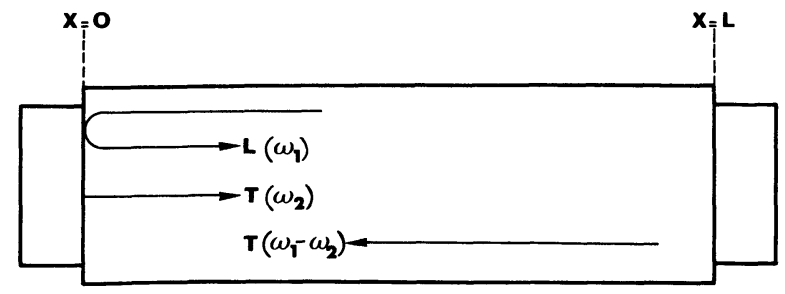

Fig. 3. - Second cas de corrélation $\left(\omega_{1}<2 \omega_{2}\right)$. [Second correlation device $\left(\omega_{1}<2 \omega_{2}\right)$.] 
Les relations (36), (45) et (47) s'écrivent cette fois :

$$
\tau_{1}=\tau_{2}=\beta=0
$$

la constante $C$ étant encore donnée par la relation (55).

L'étude du noyau de la transformation dans l'espace des fréquences s'effectue encore comme précédemment, mais comme nous avons une interaction à la fréquence différence, il convient de poser :

$$
a_{2}=-\omega_{2} .
$$

Nous constatons encore que le noyau ne diffère sensiblement de zéro pour un temps de traitement important que si la condition de synchronisme pour les ondes primaires est satisfaite.

3.3 Caractérisation Des PERformances DU SYSTÈME. - L'efficacité de l'interaction non linéaire est caractérisée par un facteur bilinéaire interne [13] $F_{\text {int }}$ reliant les densités de puissance acoustique des trois ondes interagissantes $P_{1}, P_{2}$ (ondes primaires) et $P_{3}$ (onde produite) :

$$
F_{\mathrm{int}}=P_{3} /\left(P_{1} P_{2}\right) .
$$

Afin de calculer $P_{3}$, il est nécessaire de définir une situation de référence. Nous nous plaçons pour cela au synchronisme en supposant les deux ondes primaires, transversales, modulées en amplitude par des impulsions rectangulaires de durées satisfaisant la condition de conservation (48). L'équation (22) montre alors, en calculant les densités de puissance acoustique [4] :

$$
P_{i}=\frac{\rho \omega_{i}^{2}\left|A_{i}\right|^{2}}{2\left|s_{i}\right|}
$$

et en prenant pour $P_{3}$ sa valeur maximale (au sommet du triangle obtenu en sortie), que :

$$
F_{\text {int }}=\mu\left(\omega_{\mathrm{L}} L s_{\mathrm{T}}\right)^{2}
$$

où $\omega_{\mathbf{L}}$ est la pulsation de l'onde longitudinale de sortie, $L$ la longueur du milieu d'interaction et $\mu$ un coefficient non linéaire donné, en notation contractée dans notre repère, par :

$$
\mu=\frac{\left(C_{155}+C_{11}\right)^{2} s_{\mathrm{L}}}{8 C_{11} C_{44}^{2}} .
$$

On mesure expérimentalement un facteur bilinéaire externe tenant compte de la géométrie (surface) des transducteurs piézoélectriques et de leurs efficacités.

Connaissant la largeur de bande, le facteur de bruit et le niveau de saturation du récepteur, on peut en déduire la dynamique du système. Cette grandeur peut néanmoins être limitée par des phénomènes de saturation au cours de l'interaction non linéaire à cause d'interactions parasites comme la génération d'harmoniques. D'autre part, les limites fondamentales restreignant les capacités de traitement du signal sont également fixées par un nombre de fréquences discernables dans la largeur de bande B du transducteur recevant l'onde acoustique engendrée. La relation (A.18) montre que, vu la réponse non nulle pour de petits défauts de synchronisme, on ne peut séparer que :

$$
N=B \cdot T_{\mathrm{M}}
$$

canaux de traitement opérant en parallèle, nombre augmentant avec l'acuité des conditions de synchronisme, lorsque les informations sont codées uniquement à l'aide de la modulation des ondes primaires en amplitude. Ceci restreint la largeur de bande utilisée à une valeur petite devant la fréquence des ondes. Dans le cas d'une modulation en fréquence, qui permet l'utilisation d'une largeur de bande supérieure, le nombre de points $N$ fournit une mesure de la précision de calcul.

Afin d'augmenter le nombre de points $N$ on peut, conformément à la relation (62), utiliser des fréquences opératoires supérieures, donc des largeurs de bande plus importantes, ou des milieux d'interaction plus longs, soit des temps maxima de traitement supérieurs.

Cependant, il faut tenir compte de l'atténuation des ondes acoustiques que nous avons négligée jusqu'ici. Si nous voulons qu'elle ne détériore pas sensiblement les performances du dispositif, il faut que l'atténuation des trois ondes acoustiques interagissantes soit au plus de l'ordre du décibel pour un trajet sur la longueur du milieu non linéaire. Or, l'atténuation est proportionnelle approximativement au carré de la fréquence acoustique et rigoureusement à la distance parcourue (si on l'exprime en décibels). $\mathrm{Si} \alpha_{0}$ représente le coefficient d'atténuation de l'onde longitudinale, exprimé en $\mathrm{dB} / \mathrm{cm} / \mathrm{gHz}^{2}$, et si la pulsation $\omega_{\mathrm{L}}$ est exprimée en $\mathrm{gHz}$ et la longueur $L$ en $\mathrm{cm}$, nous avons :

$$
\alpha_{0} \frac{2 \omega_{\mathrm{L}}^{2}}{3 \pi} \quad L \leqslant 1 \mathrm{~dB}
$$

pour un transducteur en niobate de lithium fournissant une largeur de bande $B$ voisine de l'octave $\left(B=\omega_{\mathrm{L}} / 3 \pi\right)$.

Les relations (60) et (62) entraînent dans ce cas la proportionnalité entre le rendement non linéaire $F_{\text {int }}$ et le carré du nombre de points $N$. Le dispositif sera optimisé vis à vis du compromis rendement non linéaire-nombre de points en utilisant un milieu de longueur maximale, compte tenu des impératifs d'homogénéité et de cristallogenèse, et une pulsation $\omega_{\mathrm{L}}$ fixée par la condition (63).

4. Etude expérimentale de processeurs paramétriques basés sur l'interaction à trois ondes. - 4.1 Caractérisation de matériauX. - Afin de définir les matériaux susceptibles d'être utilisés en acoustique non linéaire, nous avons entrepris une caractérisation 
systématique de certains, réputés pour leurs propriétés acousto-optiques $[14,15,16]$, car des arguments simples conduisent à envisager une certaine corrélation entre les efficacités acousto-optique et non linéaire acoustique des matériaux [17].

Les conditions de synchronisme (29-30) étant en général très aiguës, il est nécessaire de déterminer avec une bonne précision les vitesses des ondes acoustiques longitudinales et transversales selon certaines directions propres des matériaux précédents. Nous avons, pour cela, utilisé une sonde laser acoustooptique qui fournit l'amplitude et la phase d'un faisceau acoustique [18] continûment au cours de la propagation. En translatant la ligne acoustique devant le faisceau laser d'analyse focalisé, on peut mesurer la longueur d'onde acoustique et, connaissant la fréquence, en déduire la vitesse.

Un signal proportionnel au déplacement mécanique, délivré par un capteur de résolution égale à $1 \mu \mathrm{m}$, est appliqué à la voie $\mathrm{X}$ d'un dispositif de visualisation (enregistreur ou oscilloscope) alors que le signal de sortie du système de la figure 4 est appliqué

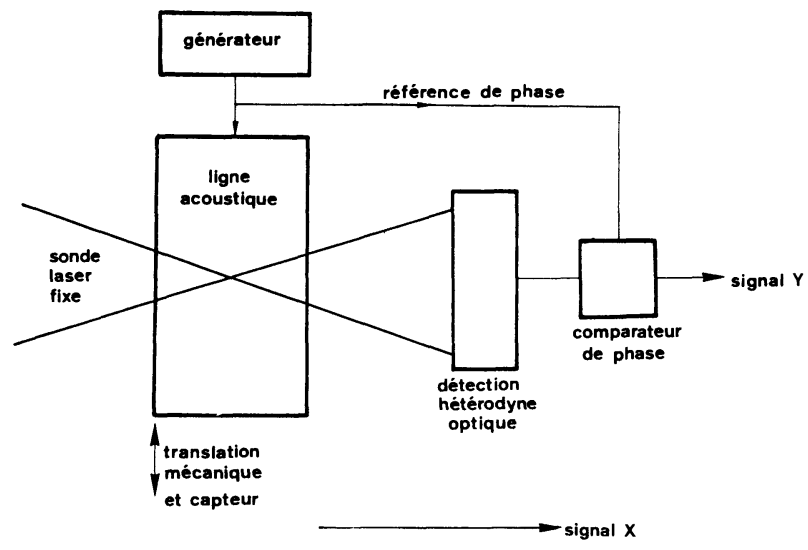

a)

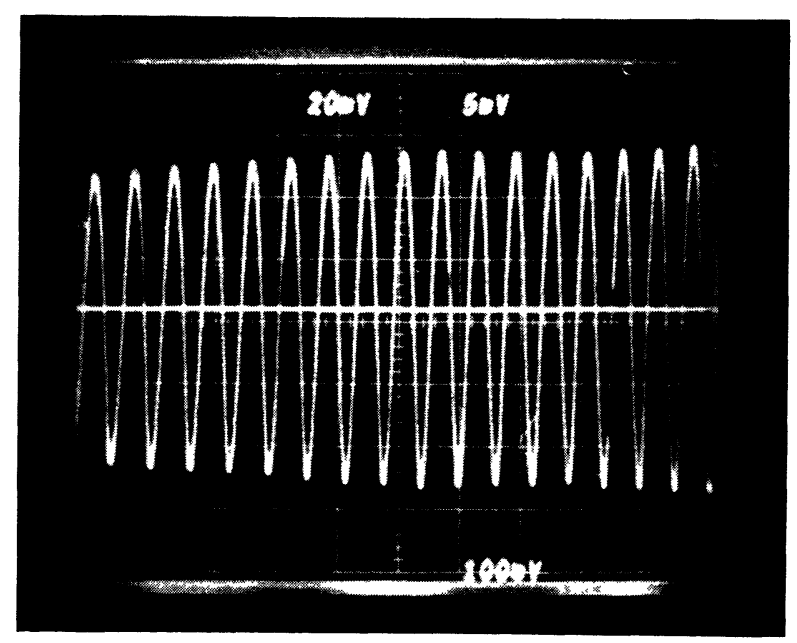

b)

Fig. 4. - Mesure de vitesse par sonde acoustique. a) Montage. b) Enregistrement typique de la phase d'une onde.

[Acoustooptic velocity measurement. $a$ ) Set-up. $b$ ) Typical result.] à la voie $\mathrm{Y}$. On peut ainsi "compter" les longueurs d'onde acoustique contenues dans une longueur déterminée du cristal et, connaissant la fréquence acoustique avec une excellente précision $\left(10^{-5}\right.$ à $\left.10^{-6}\right)$, en déduire la vitesse avec une précision voisine de $10^{-3}$ compte tenu de la linéarité du capteur et de la chaîne de réception super-hétérodyne. L'enveloppe du signal enregistré sert de plus à la détermination approximative de l'atténuation globale des ondes acoustiques, à partir de la décroissance de l'amplitude sur une distance donnée.

Nous avons également étudié la génération d'harmoniques au cours de la propagation des ondes acoustiques longitudinales [17, 18. 19], à l'aide d'une sonde acousto-optique.

L'étude de l'harmonique de rang 2 fournit la valeur du facteur de mérite non linéaire $M_{\mathrm{a}}$ avec une erreur très faible à condition d'effectuer les mesures à faible niveau de puissance en un point suffisamment éloigné du transducteur $[17,18,19]$. Ce facteur de mérite sert à l'évaluation approximative de la non-linéarité des matériaux, même si l'interaction à trois ondes utilisées fait intervenir des constantes élastiques différentes de celles décrivant la génération d'harmoniques.

4.2 Processeurs paramétriQues. - Nous avons utilisé plusieurs matériaux pour étudier les interactions paramétriques colinéaires entre ondes acoustiques de polarisations différentes. Sur les deux faces opposées du milieu ont été soudés à l'indium deux transducteurs en niobate de lithium, adaptés électriquement à large bande, l'un excitant des ondes acoustiques longitudinales et l'autre produisant des ondes acoustiques transversales.

Nous avons systématiquement vérifié expérimentalement que les conditions de synchronisme étaient toujours en parfait accord avec les prévisions théoriques déduites des valeurs des vitesses précédemment mesurées. De plus, nous avons observé toutes les possibilités des processeurs paramétriques réalisés : obtention de deux produits de corrélation et d'un produit de convolution, inversion dans le temps, fonction d'ambiguité et transformation de Fourier.

Nous donnons ici quelques photographies typiques illustrant le fonctionnement des processeurs paramétriques obtenus.

La figure 5 représente une convolution obtenue dans une ligne acoustique en molybdate de plomb, dans le cas d'ondes primaires modulées par des trains d'impulsions rectangulaires récurrentes. L'obtention d'un retard variable est illustrée à la figure 6 : le retard entre les deux impulsions modulant l'amplitude des ondes acoustiques primaires étant progressivement augmenté, le signal de convolution apparaît à des instants de plus en plus retardés. Le comportement bilinéaire des processeurs est montré à la figure 7 où l'on voit que le signal de sortie est bien proportionnel à l'un des signaux d'entrée (tout comme au second d'ailleurs). La figure 8 présente un fonctionne- 


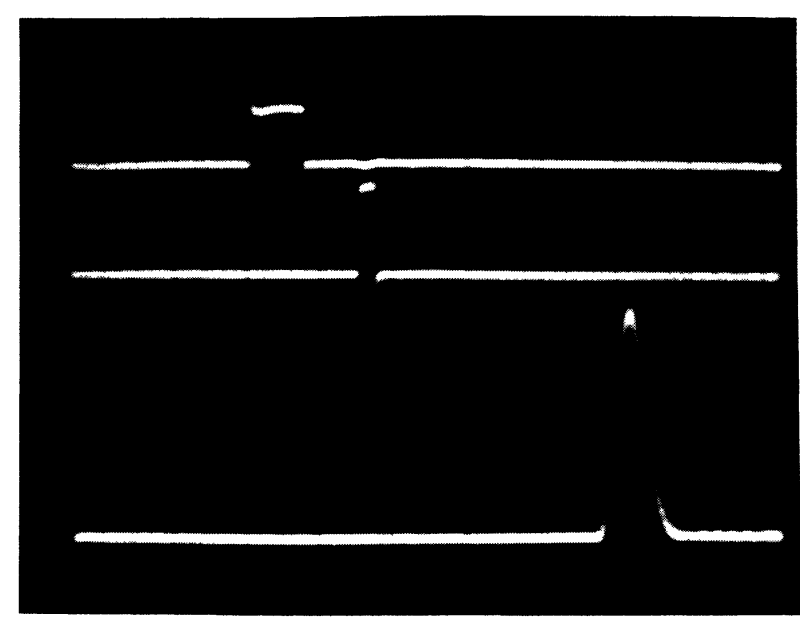

a)

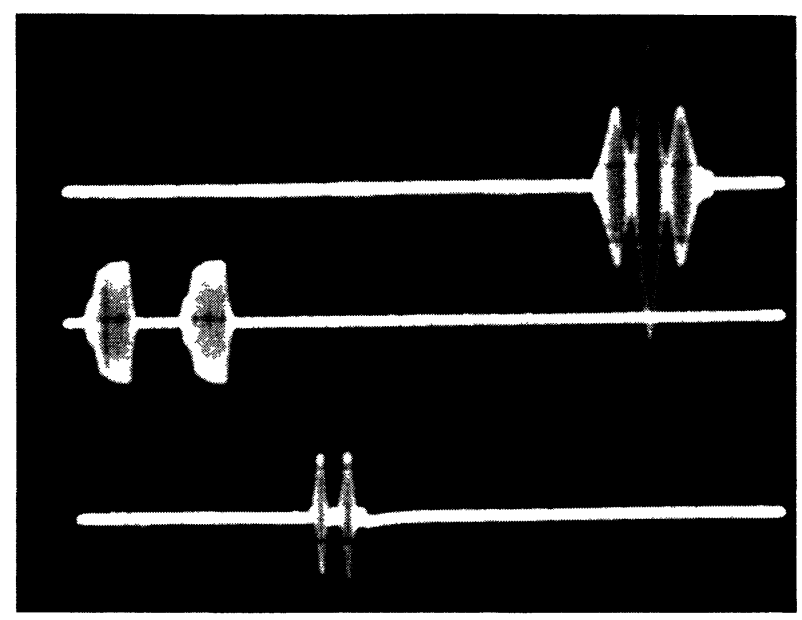

b)

Fig. 5. - Résultat typique (convolution). a) Impulsions simples. b) Impulsions doubles. Le signal le plus retardé correspond à la convolution des deux autres.

[Typical convolution result. $a$ ) Single pulses. $b$ ) Double pulses. The most time delayed signal is the convolution product of the other two.]

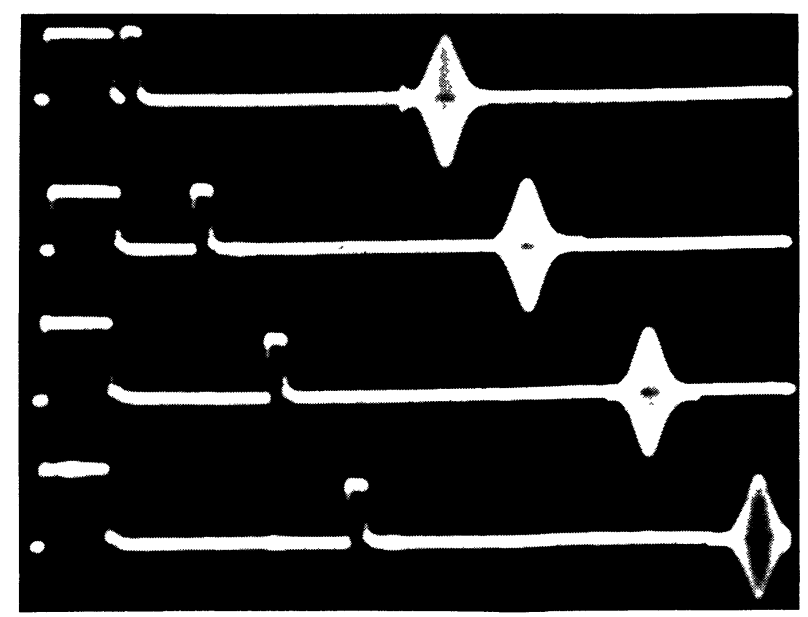

Fig. 6. - Retard variable.

[Variable time delay.]

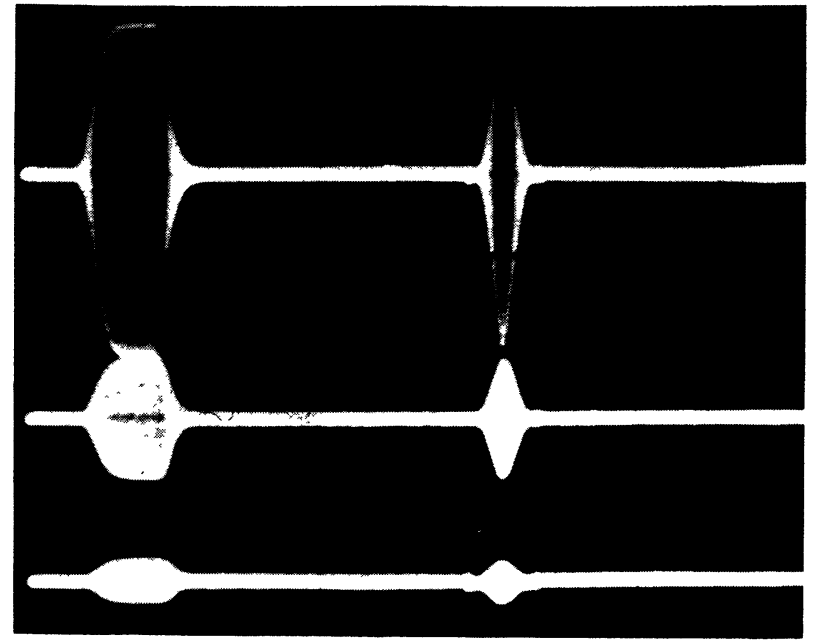

Fig. 7. - Comportement bilinéaire.

[Bilinear operation.]

ment en transformateur de Fourier pour une entrée comportant une ou plusieurs impulsions rectangulaires.

Les facteurs bilinéaires, dynamiques et nombre de points ont été étudiés.

Les principaux résultats expérimentaux sont donnés dans le tableau II pour les divers matériaux qui ont été caractérisés.

Les lenteurs, atténuations, pulsations et dynamique sont exprimées respectivement en $\mathrm{cm} / \mu \mathrm{s}, \mathrm{dB} / \mathrm{cm} / \mathrm{gHz}^{2}$, $10^{6} \mathrm{rad} / \mathrm{s}, 10^{-6} \mathrm{~W}^{-1}$ et $\mathrm{dB}$.

Le nombre de points optimum $N_{\text {opt }}$ et le rendement non linéaire optimum $F_{\text {opt }}$ sont calculés en supposant une longueur d'interaction de $2 \mathrm{~cm}$ et une pulsation $\omega_{L_{\text {opt }}}$ donnée par la condition (63).

En conclusion, on peut dire que les dispositifs de traitement du signal possédant près de 1000 points sont réalisables avec des dynamiques de l'ordre de $40 \mathrm{~dB}$ en utilisant des matériaux dont l'atténuation n'est pas prohibitive.

En sacrifiant le nombre de points, des rendements meilleurs sont obtenus et la dynamique peut devenir supérieure à $60 \mathrm{~dB}$.

5. Conclusion. - Dans le présent travail, nous avons étudié des interactions non linéaires entre ondes élastiques applicables au filtrage linéaire variable de signaux.

La fonction obtenue est une transformée bilinéaire qui se ramène, par des changements de coordonnées, à des produits de convolution et de corrélation.

Le cas simple envisagé est celui de l'interaction entre trois ondes acoustiques de volume colinéaires. L'étude complète d'un dispositif de traitement du signal basé sur ce principe a été effectuée. La possibilité de réaliser des composants à trois portes permutables à volonté, deux jouant le rôle d'entrées et la dernière celui de sortie a été démontrée. On obtient 


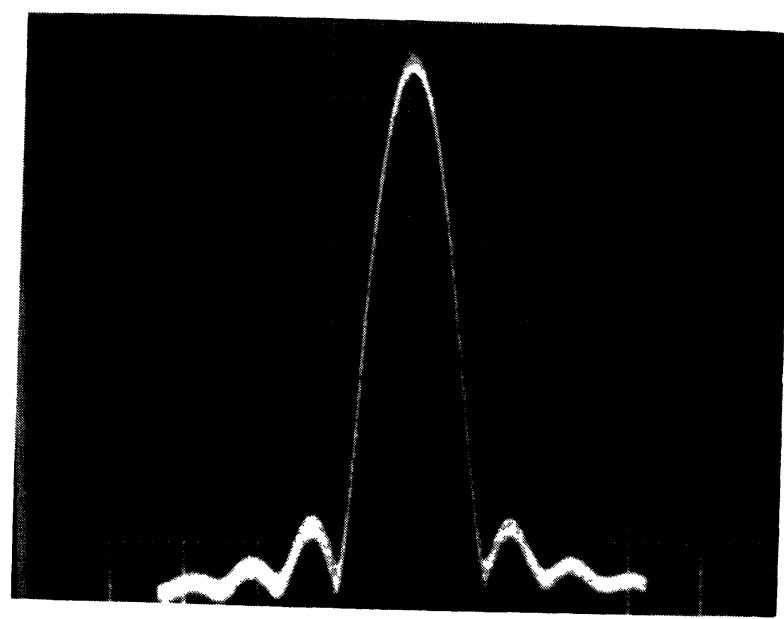

a)

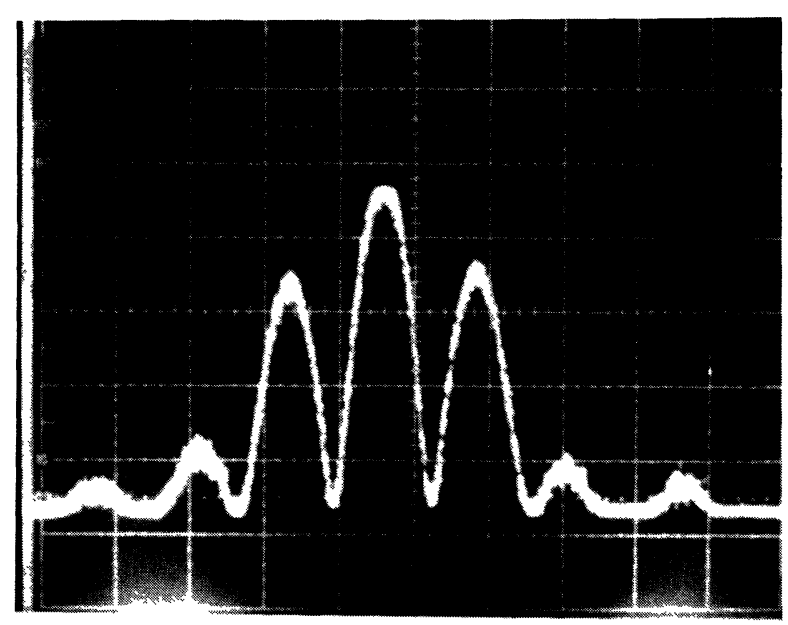

b)

Tableau II. - Résultats expérimentaux.

[Experimental results.]

$\begin{array}{ccccc}\text { Matériau } & \text { Silice } & \begin{array}{c}\text { Verre } \\ \text { SF-59 }\end{array} & \begin{array}{c}\text { Trisulfure } \\ \text { d'arsenic }\end{array} & \begin{array}{c}\text { Molybdate } \\ \text { de plomb }\end{array} \\ - & - & - & - & - \\ s_{\mathrm{L}} & 1,683 & 3,154 & 3,853 & 2,753 \\ \alpha_{0} & 20 & 3200 & 170 & 15 \\ s_{\mathrm{T}} & 2,463 & 5,435 & 7,127 & 5,102 \\ \omega_{\mathrm{L}} / 2 \pi & 200 & 25 & 50 & 250 \\ F_{\text {int }} & 15 & 15 & 800 & 1000 \\ \mu & 0,8 \times 10^{-6} & 17 \times 10^{-6} & 50 \times 10^{-6} & 12 \times 10^{-6} \\ \text { dynamique } & 55 & 55 & 65 & 80 \\ N & 250 & 70 & 220 & 120 \\ N_{\text {opt }} & 420 & 75 & 370 & 1000 \\ F_{\text {opt }} & 11 & 10 & 350 & 300 \\ \omega_{\text {opt }} / 2 \pi & 120 & 10 & 40 & 150\end{array}$

Fig. 8. - Transformée de Fourier. Signal de modulation de durée $T=2 \mu$ s consistant en : $a$ ) une impulsion rectangulaire unique ; b) deux impulsions identiques de durée $T / 3$ séparées de $T / 3$; c) trois impulsions identiques de durée $T / 5$ séparées de $T / 5$. $\mathrm{H}$ : $500 \mathrm{kHz} / \mathrm{div}$.

[Fourier transforms. The modulation signal with duration $T=2 \mu \mathrm{s}$ consists in $: a$ ) a single rectangular pulse ; $b$ ) two identical pulses with duration $T / 3$ delayed by $T / 3 ; c$ ) three identical pulses with duration $T / 5$ delayed by $T / 5$. $\mathrm{H}: 500 \mathrm{kHz} / \mathrm{div}$.] 
ainsi deux produits de corrélation à la fréquence différence de celles des deux entrées et un produit de convolution à la fréquence somme. Du point de vue expérimental, des dispositifs de performances comparables à celles des meilleurs composants acoustiques de traitement du signal actuels sont réalisables. Des dynamiques très importantes peuvent être obtenues avec un nombre de points moyen. Un compromis existe entre ces deux grandeurs du fait de l'atténuation des ondes acoustiques, ce qui permet d'obtenir un nombre de points élevé en sacrifiant un peu la dynamique. Le choix des fréquences opératoires est finalement guidé par les performances souhaitées, une fois les vitesses et atténuations des ondes acoustiques dans le matériau non linéaire utilisé connues. La caractérisation des matériaux constitue donc un aspect important de l'amélioration du type de dispositif étudié : des matériaux présentant de fortes nonlinéarités intrinsèques et des atténuations faibles sont souhaitables, impératifs souvent contradictoires. Plusieurs candidats intéressants semblent exister parmi les nouveaux matériaux acousto-optiques récemment synthétisés et qui seront commercialisés d'ici quelques années, notamment certains composés chalcogénides à base de soufre [20].

Annexe. - ETUde DES PROPRIÉTÉS DE LA TRANSFORMATION BILINÉAIRE. - Nous considérons ici une intégrale bilinéaire du type :

$$
g(z)=\int_{-\infty}^{+\infty} f(\alpha u+\beta) h(\gamma z-u) \mathrm{d} u
$$

où $\alpha, \gamma$ (facteurs d'échelle) et $\beta$ (retard) sont des coefficients constants.

L'intégrale (A.1) est une généralisation du produit de convolution mathématique (si $\gamma>0$ ) ou du produit de corrélation mathématique (autres cas). Considérons d'abord le cas idéalisé où le signal $f(x)$ est une impulsion unité de Dirac.

$$
f(x)=\delta\left(x-x_{0}\right) .
$$

Le signal de sortie obtenu s'écrit alors :

$$
g(z)=h\left(\gamma z+\frac{\beta-x_{0}}{\alpha}\right) / \alpha
$$

soit une version retardée, dilatée ou comprimée dans le temps (si $\gamma \neq 1$ ), et éventuellement inversée dans le temps $($ si $\gamma<0)$, de la réponse impulsionnelle du filtre.

Considérons maintenant l'exemple de deux impulsions rectangulaires de durées respectives $x_{1}$ et $x_{2}$ :

$$
f(x)=\left\{\begin{array}{l}
f_{1} \text { (constante) si } 0<x<x_{1} \\
0 \text { à l'extérieur de cet intervalle }
\end{array}\right.
$$

$$
h(x)=\left\{\begin{array}{l}
h_{1} \text { (constante) si } x_{\mathrm{r}}<x<x_{2}+x_{\mathrm{r}} \\
0 \text { à l'extérieur de cet intervalle }
\end{array}\right. \text { (A.5) }
$$

où $x_{\mathrm{r}}$ est un retard arbitraire (positif ou négatif).

Le signal de sortie obtenu est représenté à la figure 9.

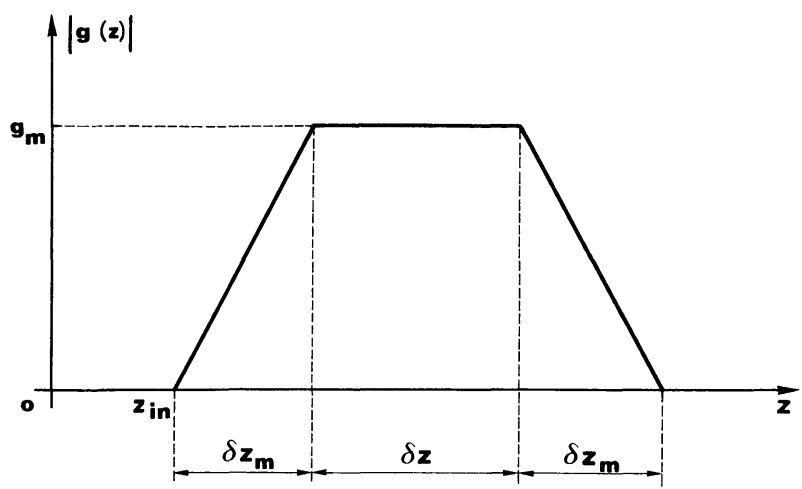

Fig. 9. - Transformée bilinéaire de deux impulsions rectangulaires.

[Bilinear transform of two rectangular impulses input signals.]

Dans cette figure, nous avons introduit les notations suivantes :

$$
\begin{aligned}
g_{\mathrm{m}} & =f_{1} h_{1} / a b s(\alpha) \\
\delta z_{\mathrm{m}} & =\min \left[x_{1}, x_{2} a b s(\alpha)\right] / a b s(\alpha \gamma) \\
z_{\mathrm{M}} & =\max \left[x_{1}, x_{2} a b s(\alpha)\right] / a b s(\alpha \gamma)
\end{aligned}
$$

et dans le cas de la convolution

$$
\begin{aligned}
& z_{\text {in }}=\left(\alpha x_{\mathrm{r}}-\beta\right) /(\alpha \gamma) \quad(\alpha>0, \gamma>0) \\
& z_{\text {in }}=\left(\alpha x_{\mathrm{r}}+x_{1}-\beta\right) /(\alpha \gamma) \quad(\alpha<0, \gamma>0)
\end{aligned}
$$

alors que pour la corrélation :

$$
\begin{aligned}
& z_{\text {in }}=\left[\alpha\left(x_{2}+x_{\mathrm{r}}\right)-\beta\right] /(\alpha \gamma) \quad(\alpha<0, \gamma<0) \quad(\mathrm{A} .11) \\
& z_{\text {in }}=\left[\alpha\left(x_{2}+x_{\mathrm{r}}\right)+x_{1}-\beta\right] /(\alpha \gamma) \quad(\alpha>0, \gamma<0) .
\end{aligned}
$$

Les variations en sens opposé de $z_{\text {in }}$ et $x_{\mathrm{r}}$ dans les deux dernières relations mettent en évidence le phénomène classique d'inversion dans le temps lié à la corrélation.

L'obtention d'un signal de sortie de forme triangulaire, et non plus trapézoïdale, est liée à la condition (correspondant à $\delta z=0$ ) :

$$
x_{1}=x_{2} a b s(\alpha)
$$

ce qui revient à dire que les échelles effectives de variation des fonctions $f$ et $h$ sont dans le rapport $a b s(\alpha)$. 
L'intégrale (A.1) peut se réécrire sous la forme générale d'un double produit de convolution [13] :

$g(z)=\iint_{-\infty}^{+\infty} k\left(u_{1}, u_{2}\right) f\left(\lambda z-u_{1}\right) h\left(\lambda z-u_{2}\right) \mathrm{d} u_{1} \mathrm{~d} u_{2}$

(A. 14)

où $k\left(u_{1}, u_{2}\right)$ représente le noyau de la transformation, donné par la fonction de Dirac :

$$
k\left(u_{1}, u_{2}\right)=\delta\left(u_{1}+\beta+\alpha u_{2}\right) / \alpha
$$

avec

$$
\lambda=\alpha \gamma /(1+\alpha) .
$$

La transformée de Fourier de l'équation (I.24) s'écrit :

$$
\begin{aligned}
G\left(a_{3}\right) & =\frac{1}{2 \pi} \iint_{-\infty}^{+\infty} \times \\
\times & K\left(a_{1}, a_{2}\right) F\left(a_{1}\right) H\left(a_{2}\right) \delta\left(a_{1}+a_{2}-a_{3}\right) \mathrm{d} a_{1} \mathrm{~d} a_{2}
\end{aligned}
$$

où $K$ est le noyau de la transformation dans l'espace de Fourier, donné par :

$$
\begin{aligned}
K & =\int_{u_{2}=0}^{A} \int_{u_{1}=-\infty}^{+\infty} k\left(u_{1}, u_{2}\right) \\
& =\frac{A}{\alpha} \exp \left[-j\left(a_{1} u_{1}+a_{2} u_{2}\right)\right] \mathrm{d} u_{1} \mathrm{~d} u_{2}
\end{aligned}
$$

où nous avons tenu compte des possibilités finies de traitement des dispositifs réels en limitant l'intégration sur $u_{2}$ à l'intervalle $[0, A]$, et introduit le paramètre :

$$
2 x_{0}=\left(a_{1} \alpha-a_{2}\right) A
$$

et le déphasage :

$$
\varphi=x_{0}+a_{1} \beta
$$

Dans le cas général, l'expression (A.18) représente l'acuité des conditions opératoires pour le dispositif de traitement.

Dans le cas idéal d'un traitement sur un intervalle infini $(A \rightarrow \infty)$, l'expression précédente tend vers une fonction de Dirac :

$$
K\left(a_{1}, a_{2}\right) \alpha \delta\left(a_{1} \alpha-a_{2}\right)
$$

ce qui redonne le facteur idéal entre les échelles de variation (A.13). Un faible écart par rapport à cette condition suffit alors pour annuler pratiquement le noyau $K\left(a_{1}, a_{2}\right)$.

Par contre, pour une valeur finie de $A$, une décroissance de moitié en valeur relative de cette fonction est approximativement obtenue pour des perturbations $\Delta a_{1}$ ou $\Delta a_{2}$ telles que $x_{0}= \pm \pi / 2$, soit :

$$
\Delta a_{2}= \pm \pi /(2 A)
$$

ou :

$$
\Delta a_{1}= \pm \pi /(2 A \alpha)
$$

Ces relations représentent les domaines pratiques d'utilisation à $-3 \mathrm{~dB}$ autour des conditions opératoires optimales.

\section{Bibliographie}

[1] Wallace, D. C., Solid State Physics, 25 (Academic) 1970, vol. 25.

[2] Thurston, R. N. et Shapiro, J. M., J. Acoust. Soc. Am. (1967), 41, 112.

[3] Musgrave, M. J. P., Crystal Acoustics (Holden Day) 1970.

[4] Auld, B. A., Acoustic Fields and Waves in Solids (Wiley) 1973.

[5] HolsKy, H., Stress Waves in Solids (Dover) 1963.

[6] Dixon, R. W., Appl. Phys. Lett. 11 (1967) 340.

[7] Rouvaen, J. M., Bridoux, E., Moriamez, M. et Torguet, R., Appl. Phys. Lett. 23 (1973) 499.

[8] Bridoux, E., Rouvaen, J. M., Haine, F. et Torguet, R. Electron. Lett. 11 (1975) 26.

[9] Bridoux, E., Rouvaen, J. M., Bruneel, C. et Torguet, R., J. Appl. Phys. 46 (1975) 2384.

[10] Brugger, K., J. Appl. Phys. 36 (1965) 759.

[11] Berkowitz, R. S., Modern Radar (Wiley) 1965.
[12] Das, P., Araghi, M. N. et Wang, W. C., Proc. I.E.E.E. 62 (1964) 1714

[13] Kino, G. S., Ludvik, S., Shaw, H. J., Shreve, W. R., White, J. M. et WinsLow, D. K., I.E.E.E. Trans. Microwave Theory Techn. MTT 21 (1973) 244.

[14] Pinnow, D. A., I.E.E.E.J. Quant. Elect. QE-6 (1970) 223.

[15] Uchida, N. et Nizeki, N., Proc. I.E.E.E. 61 (1973) 1073.

[16] Eschler, H. et Weidinger, F., J. Appl. Phys. 46 (1975) 65.

[17] Rouvaen, J. M., Bridoux, E., Moriamez, M. et Torguet, R., Appl. Phys. Lett. 25 (1974) 97.

[18] Rouvaen, J. M. et Bridoux, E., Rapport final du contrat DRME No 751076 "Systèmes Acousto-Optiques de Contrôle de Microcircuits Acoustiques " (1976).

[19] Torguet, R. et Bridoux, E., Revue Phys. Appl. 7 (1972) 291.

[20] Gottlieb, M., IsaAcs, T. J., Feichtner, J. D. and Roland, G. W., J. Appl. Phys. 45 (1974) 5145. 